\title{
Pneumococcal meningoencephalitis: evolutive particularities in a case with leukemia. Case report
}

\author{
Ioana-Raluca Todoran ${ }^{*}$, Cristina Gîrbovan² ${ }^{2}$ Carmen Chiriac ${ }^{2}$, Brînduşa țilea² ${ }^{2}$ Anca Georgescu², \\ Andreea Bodea-Alucăi ${ }^{1}$ \\ From The 10th Edition of the Scientific Days of the National Institute for Infectious Diseases "Prof Dr Matei \\ Bals" \\ Bucharest, Romania. 15-17 October 2014
}

\section{Background}

Streptococcus pneumoniae represents the main cause of meningoencephalitis in adults. Penicillin-resistant Streptococcus pneumoniae has a high prevalence, representing an important cause of mortality and morbidity.

\section{Case report}

We present the case of a female patient, aged 62 years, diagnosed with chronic lymphatic leukemia and Listeria monocytogenes meningitis in the past two years, who was hospitalized in the Clinic of Infectious Diseases I with symptoms of high grade fever, altered state of consciousness, generalized tonic-clonic seizures, right oculocephalogyric deviation and coma, Glasgow Coma Scale 3 when admitted, following intense care unit and ventilatory support for ten days.

After two days of hospitalization the patient developed a labial hemorrhagic necrotic herpetic lesion. Lumbar puncture with cerebrospinal fluid assessment revealed a turbid fluid with leukocyte count $620 / 3$ cells $/ \mu \mathrm{L}$, glucose $19 \mathrm{mg} / \mathrm{dL}$, Pandy reaction was positive. Blood count interpretation indicated leukocytosis 14,000 cells $/ \mathrm{cmm}$, lymphocytosis $70 \%$ and neutropenia $23 \%$. Cerebrospinal fluid Gram strains reveals Gram-positive diplococci and CSF culture grew penicillin-resistant Streptococcus pneumoniae. Computer tomography of the brain was negative for an acute intracranial pathology, and showed fluid collections and mucosa thickening of maxillary sinus.

Treatment was promptly administered with meropenem, vancomycin, corticosteroids, acyclovir and intensive care

* Correspondence: itodoran@yahoo.com

${ }^{1}$ Clinic of Infectious Diseases I, Tîrgu Mureş, Romania

Full list of author information is available at the end of the article support led to patient survival, with a long, but favorable evolution of three weeks.

\section{Conclusion}

Pneumococcal meningoencephalitis is a severe disease with high incidence in immunocompromised patients, which can lead to systemic complications in patients aged 60 years and older. A promptly administered treatment with broad-spectrum antibiotics is necessary, since resistance to penicillin of Streptococcus pneumoniae is common. Clinical evolution of patient was favorable, despite the multiple comorbidities and the profoundly altered general condition on admission and a low score on the Glasgow coma scale.

\section{Consent}

Written informed consent was obtained from the patient for publication of this Case report and any accompanying images. A copy of the written consent is available for review by the Editor of this journal.

\section{Authors' details}

${ }^{1}$ Clinic of Infectious Diseases I, Tîrgu Mureș, Romania. ${ }^{2}$ University of Medicine and Pharmacy Tîrgu Mureş, Romania.

Published: 15 October 2014

doi:10.1186/1471-2334-14-S7-P94

Cite this article as: Todoran et al: Pneumococcal meningoencephalitis: evolutive particularities in a case with leukemia. Case report. BMC Infectious Diseases 2014 14(Suppl 7):P94. 